\title{
Quasi-isochronous triple-bend achromat with periodic stable optics and negligible coherent-synchrotron-radiation effects
}

\author{
Chengyi Zhang® and Yi Jiao๑* \\ Key Laboratory of Particle Acceleration Physics and Technology, Institute of High Energy Physics, \\ Chinese Academy of Sciences, Beijing 100049, China and University of Chinese Academy of Sciences, \\ Beijing 100049, China \\ Cheng-Ying Tsai॰ \\ Department of Electrotechnical Theory and Advanced Electromagnetic Technology, \\ Huazhong University of Science and Technology, Wuhan, 430074, China
}

(Received 5 February 2021; accepted 8 June 2021; published 24 June 2021)

\begin{abstract}
In modern high-brightness electron accelerators, the goal of preserving the beam quality from the source has long been pursued to ensure the fulfillment of their scientific missions. However, when delivering the electron beam through a beam transfer line, which may consist of bending magnets, the performance of a high-brightness electron beam may be degraded by the coherent synchrotron radiation (CSR) and the residual longitudinal dispersion. It is of critical importance to preserve the transverse emittance and control the bunch length variation as well as maintain the longitudinal phase space distribution in such a beam transfer line. For a multipass beam transfer line, the achromatic cell designs with stable optics would bring great convenience. In this study, for the periodic stable symmetric triple-bend achromat designs with identical dipoles, it is discovered that the CSR-induced emittance growth and the longitudinal dispersion up to high orders can be minimized simultaneously when a certain transfer matrix relation is satisfied; to be specific, the matrix entries between the first two dipoles should follow $\left[m_{11}, m_{21}\left(\mathrm{~m}^{-1}\right)\right] \approx(-2,0)$. Numerical optimizations demonstrate that the normalized transverse emittance, the bunch length, and the longitudinal phase space distribution are well preserved. In addition, such a design strategy shows the potential merit of effectively mitigating the microbunching instability.
\end{abstract}

DOI: 10.1103/PhysRevAccelBeams.24.060701

\section{INTRODUCTION}

The ever-increasing user demands for modern electron accelerators require high-brightness beams for higher luminosity of colliders [1-3], higher photon brightness of synchrotron-radiation storage rings and free-electron lasers (FELs) [4-10]. Typical parameters of the electron beams include the sub-picosecond (sub-ps) bunch length, a few hundreds to kilo-ampere (kA) peak current, and micrometer $(\mu \mathrm{m})$ or sub- $\mu \mathrm{m}$ normalized transverse emittances. Generation and transport of such high-brightness beams with minimum phase space quality degradation is fundamental for the design and operation of modern accelerators, so as to avoid any possible decline of the machine performance. The beam transfer line usually

\footnotetext{
*Corresponding author. jiaoyi@ihep.ac.cn

Published by the American Physical Society under the terms of the Creative Commons Attribution 4.0 International license. Further distribution of this work must maintain attribution to the author(s) and the published article's title, journal citation, and DOI.
}

consists of multiple bends for beam manipulations. For instance, in FEL spreaders, dipole magnets are often used to deflect the trajectory and deliver electron beams to several undulators for multiuser beam line operation in a FEL switchyard [4,9-11]. Preservation of transverse emittance, bunch length, as well as uniformity of the bunch current profile through a transfer line is highly desirable in FEL applications [12].

However, when such a high-brightness bunch passes through the dipole magnets of a transfer line, coherent synchrotron radiation (CSR) and longitudinal dispersion can have a significant impact on beam dynamics. These effects require careful consideration or else they may spoil the beam quality both transversely and longitudinally [13]. When relativistic beam traverses a bending magnet, CSR can be emitted if the radiation wavelength is comparable to the bunch length scale and the bunch charge density is modulated at these wavelengths [14]. The energy deviation caused by CSR brings about dilution of the transverse emittance in the dispersive bending system [15].

In the longitudinal plane, the CSR effect can give rise to the so-called microbunching instability (MBI) [16], as a small initial density modulation can convert to an energy 
modulation due to CSR, and the energy modulation may in turn be transformed back to the density modulation in the dispersive region of the transfer line. When forming a positive feedback, the density-energy conversion will lead to enhancement of the phase space modulations, and in some serious cases, induce the MBI, which has been known as the CSR-induced MBI [17].

Besides, the longitudinal dispersion effect can also influence the longitudinal dynamics, since in the dipole magnet, the particle's momentum deviation $\delta$ is correlated to the longitudinal bunch coordinate $z$ via $R_{56}, T_{566}, U_{5666}$, etc., which are often referred to as the longitudinal dispersion functions [18]. The transfer map for the longitudinal coordinate from the beam line entrance (denoted with subscript $i$ ) to the exit (denoted with subscript $f$ ) can be expressed as

$$
z_{f}=z_{i}+R_{56} \delta_{i}+T_{566} \delta_{i}^{2}+U_{5666} \delta_{i}^{3}+O\left(\delta_{i}^{4}\right) .
$$

This kind of correlation causes deviation of the final longitudinal position from the center of the bunch. For the FEL beams with an energy chirp [i.e., $\mathrm{d} E /(E \mathrm{~d} z)$ ], this correlation may result in undesirable bunch length variation, e.g., generating a longer bunch which may cause reduction of the temporal coherence and an increase in the FEL bandwidth [19] or further compressing the bunch which may have the risk of enhancing collective effects like the CSR effect, etc. This kind of $z-\delta$ correlation may become rather severe for bunches with large $\delta$. One can see that, in the beam transfer line design, it is of vital importance to mitigate the CSR effect and the longitudinal dispersion effect simultaneously in order to maintain high beam quality. Although the additional energy deviation caused by CSR is unavoidable, the CSR-induced emittance growth and the longitudinal dispersion functions, i.e., $R_{56}$, $T_{566}, U_{5666}$, etc., could be minimized.

It has been shown that the CSR effect and the longitudinal dispersion effect can be minimized through manipulation of beam optics. In the aspect of mitigating the CSR-induced emittance growth, the cell-to-cell phase matching method was proposed by Douglas and Di Mitri to compensate for the CSR kicks with optics symmetry [2023]. Besides, the beam envelope matching method was proposed by Hajima [24] to minimize the projected emittance growth by adjusting the Twiss functions, thereby making the unperturbed beam ellipse at the exit of the beam line align with the direction of the CSR kick. The CSR kick model proposed by Jiao [25] provides a way to analyze the net CSR-induced coordinate deviations exerted by one dipole (see the Appendix B for the details), and the generic conditions for suppressing the CSR-induced emittance growth were identified for a double-bend achromat and triple-bend achromat (TBA) cell $[25,26]$. In view of the longitudinal dispersion effect, isochronicity is a sufficient condition for an achromatic system, but not a necessary condition [12]. Canceling $R_{56}$ is achievable by incorporating dipoles and quadrupoles. In our study, necessary adjustments are made (e.g., adjusting the position of dipoles and quadrupoles and the strengths of quadrupoles) to ensure that the transverse dispersion function at the center of the lattice is negative for the convenience of meeting the isochronous condition. The correction of $T_{566}$ is commonly carried out using the sextupoles $[12,18,27,28]$, which may also generate geometrical aberrations. Besides, $T_{566}$ is also affected by dipoles and quadrupoles in the lattice [18]. As for $U_{5666}$, octupoles may help to reduce the $U_{5666}$ term $[29,30]$. In regard to mitigation of CSR-induced MBI, schemes have been intensely studied [31-35], and the sufficient conditions for effective suppression of CSR-induced MBI in recirculating accelerators and single-pass facilities which may consist of multidipole transport lines were concluded [14] as: (i) small beta functions within the dipoles; (ii) the phase advance between two dipoles in a two-dipole system is close to integer times of $\pi$.

The TBA structure is regarded as one of the basic configurations to make an isochronous cell and a relatively simple structure (one can also use multibend lattice design to realize an isochronous cell, however, this is beyond the scope of this article). TBA has been widely used to transport an ultrashort, high-brightness electron beam $[29,30,36]$. In one of the previous relevant works of the TBA designs, Huang et al. [26] derived the cancellation condition of the CSR-induced emittance growth using the CSR kick model. Such a cancellation condition requires that a certain form of the transverse transfer matrix between the center of two dipoles should be satisfied. Besides, using the method similar to Ref. [25], it has been demonstrated [37-39] that a kind of TBA design could yield both the first-order isochronicity, i.e., $R_{56}=0$ and the steady-state-CSR-induced emittance growth canceled by requiring, however, somewhat extreme constrains of the transfer matrix such that the trace of the total transverse transfer matrix exceeds 2 in such TBA design, which means it fails to satisfy the stability for a cell with periodic stable optics. As a result, such a design may be suitable for a single-pass situation instead of a multipass situation. It would bring great convenience for the beam transfer line design targeted for multipass facilities if the TBA cell has periodic stable optics, and simultaneously be capable of maintaining the beam distribution as much as possible in 6D space.

We may deserve to summarize the main contributions of this work here. First, for a periodic stable symmetric TBA with identical bending magnets, we find a condition that can theoretically cancel $R_{56}$ and CSR-induced emittance growth, which is different from Ref. [39]. The condition we find has an extra potential merit of mitigating the MBI. Second, such TBA cell designs are obtained through the multiobjective particle swarm optimization (MOPSO) method, the characters of which verify the theoretical 
analysis. Third, using the MOPSO method, the high-order longitudinal dispersion effects are further suppressed so that such TBA cell designs are isochronous up to high orders, thus better maintaining the beam quality. These designs can be useful for a beam with large energy spread, e.g., the beam from the laser-plasma accelerators (LPAs).

The paper is organized as follows. In Sec. II, the theoretical analysis of minimizing $R_{56}$ and the CSRinduced emittance growth for a periodic stable TBA and the derivation of the condition are presented. Then practical TBA designs are obtained using the MOPSO method, the characters of which verify the analysis. Section III is dedicated to the optimization of high-order longitudinal dispersion functions and simulations of multiunit cells with practical considerations. The TBA designs being approximately CSR-immune, isochronous up to high orders and free from MBI are found. The conclusion and discussion are presented in Sec. IV.

\section{THEORETICAL ANALYSIS AND NUMERICAL VERIFICATION}

\section{A. Theoretical analysis}

Here we consider a midpoint symmetric TBA structure that consists of three identical dipole magnets. The schematic and denotation of the transfer matrices are shown in Fig. 1. In the hard-edge model of dipole magnets, the small bend-angle approximation is adopted and the lowest order of the Taylor expansion with respect to $\theta$ is used in the expression of the dipole transfer matrix, similar to the treatment of Ref. [39]. For simplicity, here we only consider the transverse motion in the horizontal plane and the longitudinal motion of the particle. The transfer matrix for the phase-space coordinate vector $\left(x, x^{\prime}, z, \delta\right)$ is used, which is part of the typical $6 \times 6$ transfer matrix that describes the motion of $\left(x, x^{\prime}, y, y^{\prime}, z, \delta\right)$, though we still adopt the subscripts 5 and 6 for the longitudinal dispersion functions $R_{56}, T_{566}$ and $U_{5666}$. The transfer matrix of a small-angle sector dipole can be expressed by

$$
R_{B}=\left(\begin{array}{cccc}
1 & L_{B} & 0 & L_{B} \theta / 2 \\
0 & 1 & 0 & \theta \\
\theta & L_{B} \theta / 2 & 1 & L_{B} \theta^{2} / 6 \\
0 & 0 & 0 & 1
\end{array}\right)
$$

where in the following discussion $L_{B}$ and $\theta$ are given quantities. Without loss of generality, the transfer matrix from the first dipole's exit to the second dipole's entrance can be expressed by

$$
M=\left(\begin{array}{cccc}
m_{11} & m_{12} & 0 & 0 \\
m_{21} & m_{22} & 0 & 0 \\
0 & 0 & 1 & 0 \\
0 & 0 & 0 & 1
\end{array}\right) .
$$

The length of the drift space at the entrance of the TBA is $d$, which is the only free parameter in $R_{d}$.

Here we focus on the relation between the four entries $m_{11}, m_{12}, m_{21}$ and $m_{22}$. The total transfer matrix of the TBA cell is

$$
M_{\mathrm{tot}}=R_{d} R_{B} \hat{M} R_{B} M R_{B} R_{d}
$$

where $\hat{M}$ has the same entries as $M$, except that $m_{11}$ and $m_{22}$ are exchanged.

First, the symplecticity and the achromatic condition are used, thus eliminating two entries among $m_{11}, m_{12}, m_{21}$ and $m_{22}$ (see Appendix A for the details). Then only two elements, say $m_{11}$ and $m_{21}$, are independent. The other two dependent elements, i.e., $m_{12}$ and $m_{22}$ can be expressed as

$$
\begin{aligned}
& m_{12}=\frac{-2-m_{11}-L_{B} m_{11} m_{21}}{2 m_{21}}, \\
& m_{22}=\frac{1}{2}\left(-1-L_{B} m_{21}\right) .
\end{aligned}
$$

Then, the stability criterion for periodic stable optics in the $x$ plane, $\left|\left(M_{\text {tot }}\right)_{11}+\left(M_{\text {tot }}\right)_{22}\right| \leq 2$, will occupy an area in

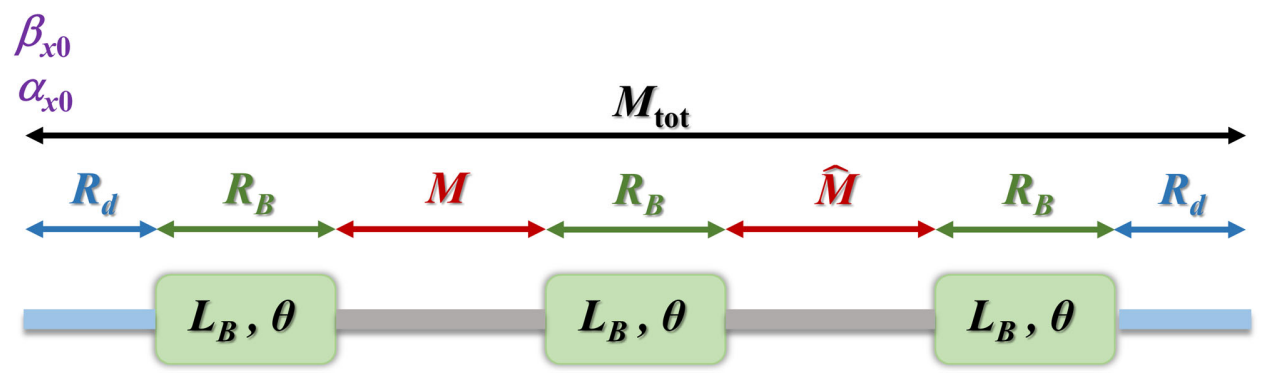

FIG. 1. Schematic of the TBA structure with three identical dipole magnets of length $L_{B}$ and bending angle $\theta . R_{d}$ and $R_{B}$ represent the transfer matrix of the drift space and the dipole, respectively. The elements in the section between the adjacent dipoles are assumed as nondipole elements, e.g., quadrupoles, sextupoles and drifts, the transfer matrices of which are expressed as $M$ and $\hat{M}$. The total transfer matrix is denoted as $M_{\mathrm{tot}}$ and $\beta_{x 0}, \alpha_{x 0}$ are the horizontal Twiss functions at the entrance of the TBA. 
the $m_{11}$ and $m_{21}$ space, which will be referred to as the stable area in what follows.

To study the requirements of canceling $R_{56}$ and the CSRinduced emittance growth, $\Delta \varepsilon_{n}$, the two conditions will be imposed separately, i.e., the first-order isochronous condition $\left(R_{56}=0\right)$ and the cancellation condition of the CSRinduced emittance growth $\left(\Delta \varepsilon_{n}=0\right)$. The former can be analyzed directly by matrix multiplication. The latter will be investigated using the CSR kick model $[25,26]$ (see Appendix B). With either constraint imposed, one of $m_{11}$ and $m_{21}$ can be eliminated, and the three dependent variables can now be expressed by the remaining one free variable. We choose $m_{11}$ as the free variable, and focus on the relation between $m_{11}$ and $m_{21}$. The condition $R_{56}=0$ requires (see Appendix A)

$$
m_{21}=\frac{2\left(2+m_{11}\right)}{L_{B}} .
$$

Cancellation of the CSR-induced emittance growth calls for (see Appendix B)

$$
m_{21}=\frac{5-2 m_{11} \pm \sqrt{121+28 m_{11}+4 m_{11}^{2}}}{2 L_{B}} .
$$

The expressions in Eqs. (6) and (7) determine two sets of requirements of $m_{21}$ as a function of $m_{11}$. The cross points of the two requirements suggest complete removal of both $R_{56}$ the CSR-induced emittance growth. The two cross points are located at

$$
\begin{gathered}
m_{11}=\frac{7}{4}, \quad m_{21}=\frac{15}{2 L_{B}}, \quad[\text { Cross point } \mathrm{A}] \\
m_{11}=-2, \quad m_{21}=0 . \quad[\text { Cross point } \mathrm{B}]
\end{gathered}
$$

The two points, together with the optics stable region, are shown in Fig. 2, where we take $L_{B}=0.4 \mathrm{~m}, \theta=4^{\circ}$ and $d=0.6 \mathrm{~m}$ as an example. The cross point $\mathrm{A}$ is the result derived in Ref. [39]. Here we find the other cross point B, which is exactly on the edge of the stable area and is independent of $L_{B}, \theta$ and $d$. Combing Eqs. (5)-(7), we find that the cross point B is a singular point. Despite the fact that the point B satisfies the optics stability, i.e., $\left|\left(M_{\text {tot }}\right)_{11}+\left(M_{\text {tot }}\right)_{22}\right|=2$, the periodic solution of the horizontal beta function at the entrance of a TBA design, $\beta_{x 0}$, will diverge [see Fig. 3(a)]. The explicit expressions for the periodic Twiss functions are given in Appendix C.

From the above discussion, we are forced to strike a compromise between reaching the isochronous condition and cancellation of CSR-induced emittance growth. It thus becomes finding a minimized $R_{56}$ at the exit of the beam line and $\Delta \varepsilon_{n}$ in the vicinity of the cross point $\mathrm{B}$, knowing that the two requirements cannot be completely satisfied in the stable area. Our observation indicates that both the

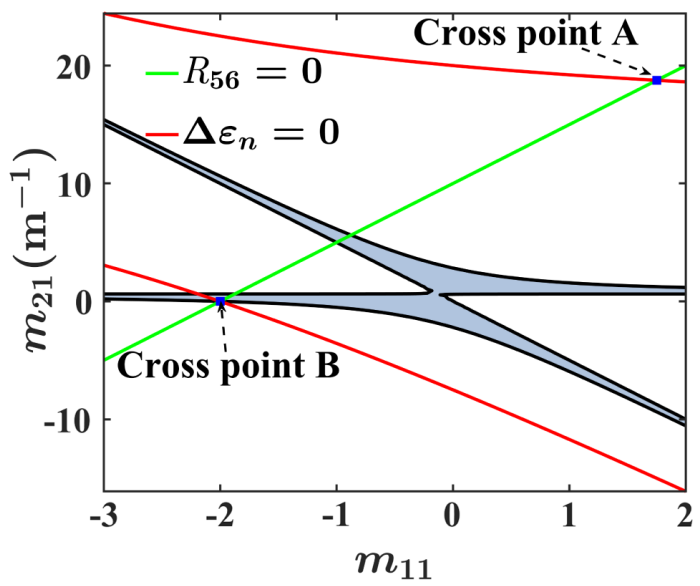

FIG. 2. The stable area (shaded) and the requirements of $m_{21}$ as a function of $m_{11}$ derived from the imposed constraint of $R_{56}=0$ (green line) and $\Delta \varepsilon_{n}=0$ (red line). The cross points are marked (blue square).

minimized $R_{56}$ and $\Delta \varepsilon_{n}$ can be found in the vicinity of the singular point, as shown in Figs. 3(b) and 3(c). Although the point $\mathrm{B}$ cannot be chosen as a suitable solution for the setting of TBA design, it allows us to find satisfactory solutions near the singular point. Moreover, we find the phase advance between the adjacent dipoles of the symmetric structure, $\Delta \psi$, is close to $\pi$, which satisfies one of the sufficient conditions [14] for effective suppression of CSR-induced MBI. It suggests an extra possible merit of mitigating the MBI.

\section{B. Numerical verification}

To verify the analysis, a simple TBA cell is designed with practical considerations (e.g., quadrupoles and sextupoles are added for dispersion matching and chromaticity correction, respectively), as shown in Fig. 4. The MOPSO [40] method is adopted in our optimization. This algorithm provides a robust way to optimize a physical problem by iteratively improving the candidate solutions based on the quality of the "fitness value," which is also referred to as the "objective function." Each candidate solution possesses a position in $n$-dimensional space defined by $n$ variables. By adjusting the variables within a certain range, the candidate solutions are updated iteratively in searching for optima [41-44]. MOPSO method is suitable to handle multiobjective optimization problems and has been widely used in accelerator optimization problems (see, e.g., [45-48]).

In the construction of the TBA cell, for the dipole magnet we set $L_{B}=0.4 \mathrm{~m}, \theta=4^{\circ}$, which corresponds to the values in the theoretical analysis. The drift length $d$ is fixed at $0.6 \mathrm{~m}$ for the convenience of comparison. The center-symmetric structure with three identical dipoles provides a 12-degree horizontal bending. The quadrupoles are grouped into three different families, one family of 

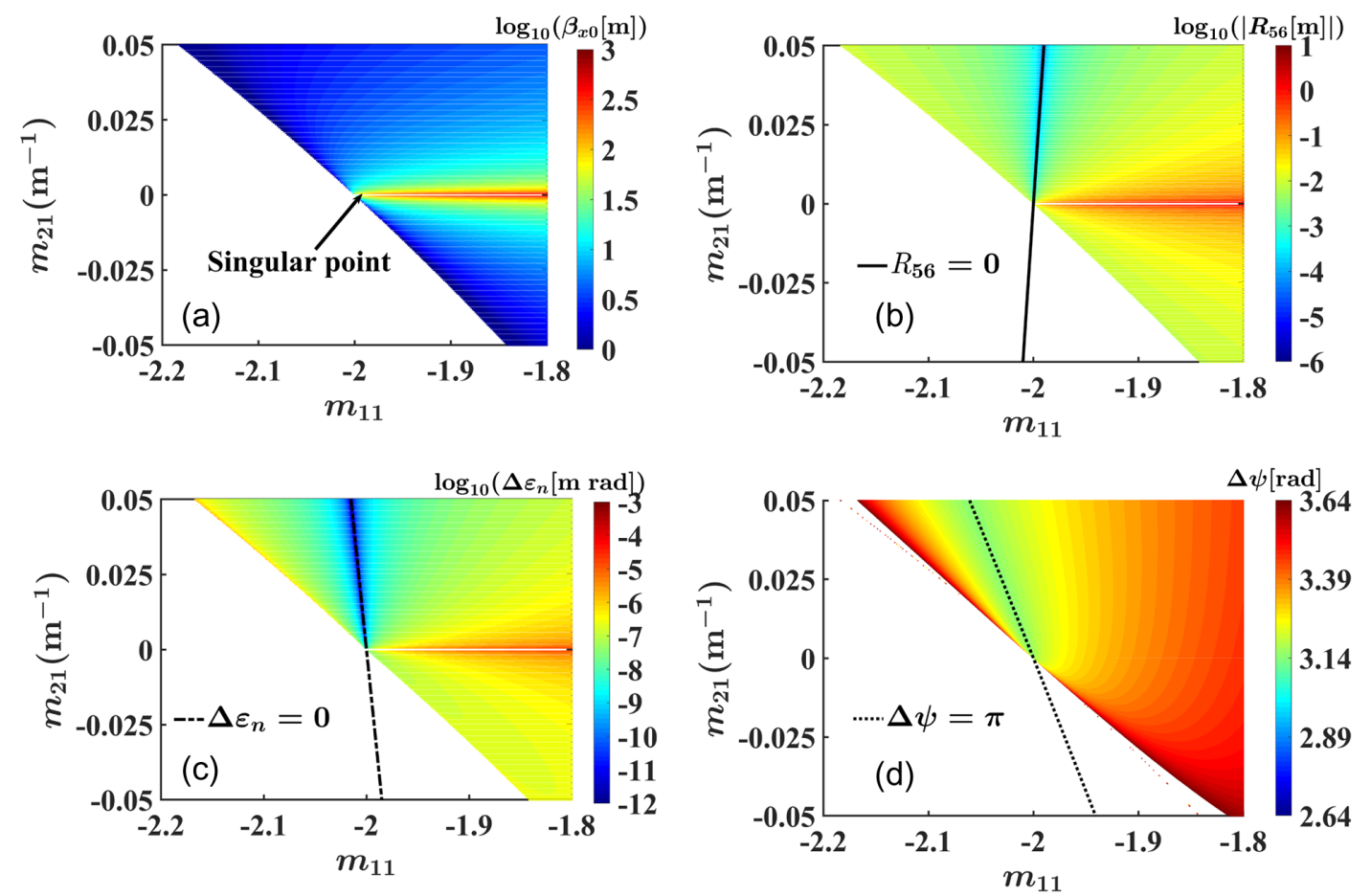

FIG. 3. (a) The horizontal beta function at the start point of the TBA, $\beta_{x 0}$. (b) The value of $R_{56}$ (the solid line shows $R_{56}=0$ ). (c) The value of CSR-induced emittance growth (the chain-dotted line shows $\Delta \varepsilon_{n}=0$ ). (d) The value of the phase advance between the adjacent dipoles (the dotted line shows $\Delta \psi=\pi$ ).

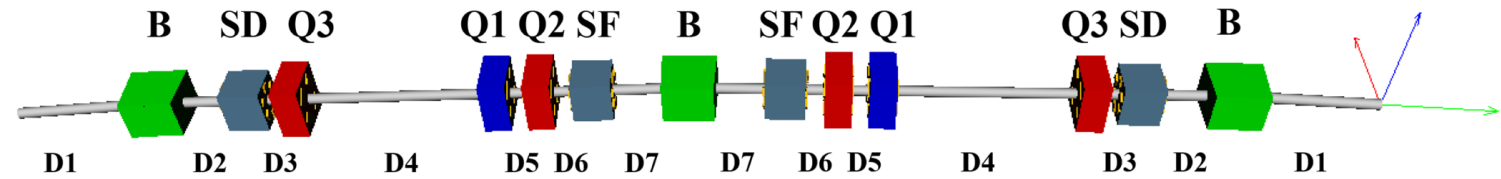

FIG. 4. The lattice structure of TBA design and the magnet layout. The green cuboids represent dipoles, the red ones and the blue ones represent focusing quadrupoles and defocusing quadrupoles, respectively, while the indigo blue ones represent sextupoles.

which is used for dispersion matching. Moreover, two families of sextupoles are used to correct the chromaticity.

There is a total of eight free variables, among which six are the drift lengths and the remaining two variables are the strengths of the quadrupoles. They can be adjusted within the preset ranges, e.g., the drift length ranges from 0.1 to $2 \mathrm{~m}$ and the maximum strength of the quadrupoles is $30 \mathrm{~m}^{-2}$. Two objective functions are set to be the weighted first order longitudinal dispersion $\alpha_{1}$ and the weighted normalized CSR-induced emittance growth $\Delta \varepsilon_{n}$ obtained from the simulation result of ELEGANT [49]. Here we point out that, typically in the literature of storage ring lattice design, the momentum compaction factor of high orders is used to describe the relative increase in closed-orbit length $C$ for a particle with the momentum deviation $\delta$, specifically $\Delta C / C_{0}=\sum_{i=1}^{\infty} \alpha_{i} \delta^{i}$, where $C_{0}$ is the on-momentum closed-orbit length [50]. In the beam transfer line, however, the transfer map for the longitudinal coordinate from the linac entrance to the exit is usually described by the longitudinal dispersion functions [see Eq. (1)]. The two conventions can be related by $R_{56}=\alpha_{1} z_{0}, T_{566}=\alpha_{2} z_{0}$, and $U_{5666}=\alpha_{3} z_{0}$, where $z_{0}$ is the trajectory length of reference particle which is a constant for a certain lattice. The matrix elements $\left(R_{56}, T_{566}\right.$ and $\left.U_{5666}\right)$ and momentum compaction factors $\left(\alpha_{1}, \alpha_{2}\right.$ and $\left.\alpha_{3}\right)$ are considered to be equivalent. In the ELEGANT simulation, the conventional rf beam parameters listed in Table I are used as the initial beam parameters. The simulation of CSR-induced emittance growth considers the transient CSR at the entrance of a

TABLE I. Parameters of the ELEGANT simulation for conventional rf beams.

\begin{tabular}{lll}
\hline \hline Parameters & Value & Unit \\
\hline Bunch length & 30 & $\mu \mathrm{m}$ \\
Beam energy & 1000 & $\mathrm{MeV}$ \\
Normalized emittance & 2 & $\mu \mathrm{m} \mathrm{rad}$ \\
Bunch charge & 500 & $\mathrm{pC}$ \\
Relative rms energy spread & 0.5 & $\%$ \\
Momentum chirp & 16.5 & $\mathrm{~m}^{-1}$ \\
\hline \hline
\end{tabular}




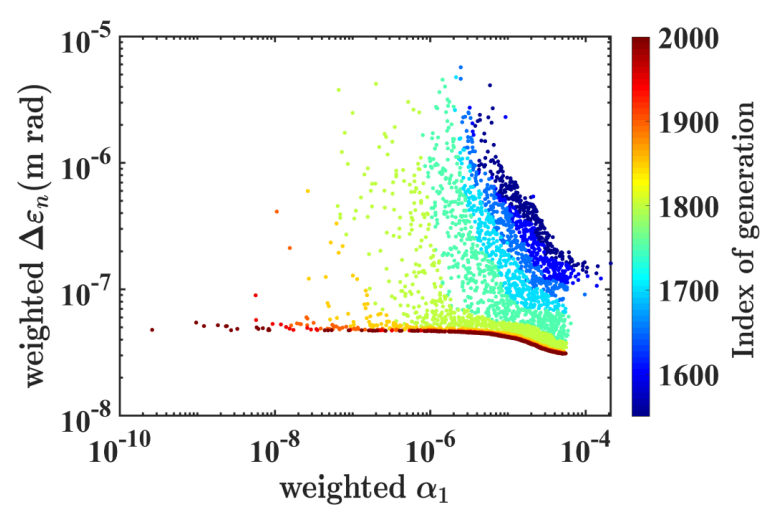

FIG. 5. Objective functions of the last 500 generations.

bending dipole and the CSR in the drift spaces following the dipoles.

To construct an isochronous, CSR-immune and stable TBA lattice, a set of initial seeds with periodic stable optics are generated and ensuring that they are fairly distributed in the stable area, as can be seen in Fig. 6(a). One family of quadrupoles is tuned to realize the achromatic condition and two families of sextupoles are used to correct chromaticity before evaluation of the two objective functions for each ensemble of variables. The optimization is conducted with practical considerations by imposing constraints. For example, the horizontal and vertical beta functions are controlled to an acceptable range of 0.1 to $1000 \mathrm{~m}$. If any one of the constraints is violated during the optimization process, the objective functions are multiplied by a factor larger than 1, which is referred to as the "weight factor." These weight factors serve as extra criteria to measure the quality of the objective functions to meet our optimization constraints.

After evolving 2000 generations, the results have become convergent. The last 500 generations are presented in Fig. 5. The distribution of $\left[m_{11}, m_{21}\left(\mathrm{~m}^{-1}\right)\right]$ of the 2000th generation is shown in comparison with the distribution of the initial seeds in Fig. 6(a). As can be seen in the figure, the solutions mainly distribute in the neighborhood of the singular point. Especially for the value of $m_{11}$, the maximum difference between the optimized results and the theoretical prediction value, $m_{11}=-2$, is less than 0.07 [see Fig. 6(b)]. It shows that the solutions with both of the minimized objective functions are found in the area between the two curves of $R_{56}=0$ and $\Delta \varepsilon_{n}=0$, which is in accordance with our theoretical analysis.

\section{OPTIMIZATION OF HIGH-ORDER LONGITUDINAL DISPERSION FUNCTIONS AND SIMULATIONS WITH PRACTICAL CONSIDERATIONS}

\section{A. Optimization results}

It is desirable for the isochronous TBA cell design if the residual high-order longitudinal dispersion functions, such as $\alpha_{2}, \alpha_{3}$ (or $T_{566}, U_{5666}$ ) and even higher orders, can be suppressed. A fully isochronous beam transport can be in demand, especially for the FEL injection line, which may need to accommodate a large beam energy spread [12]. In this situation, theoretical formulation of the $n$th order isochronous condition and the associated explicit cancellation method with respect to the $n$th order longitudinal dispersion functions would become much more involved than that presented in this paper. However, these high order terms of longitudinal dispersion function can be investigated conveniently via numerical optimizations and therefore it enables a possibility to minimize the high-order longitudinal dispersion functions and the CSR-induced emittance growth simultaneously by further optimizing currently proposed TBA design.

With similar settings of the optimization, the additional modification here is that the contribution of the residual high-order part of the longitudinal dispersion functions aside from $R_{56} \delta$ is now optimized as a whole by adding a
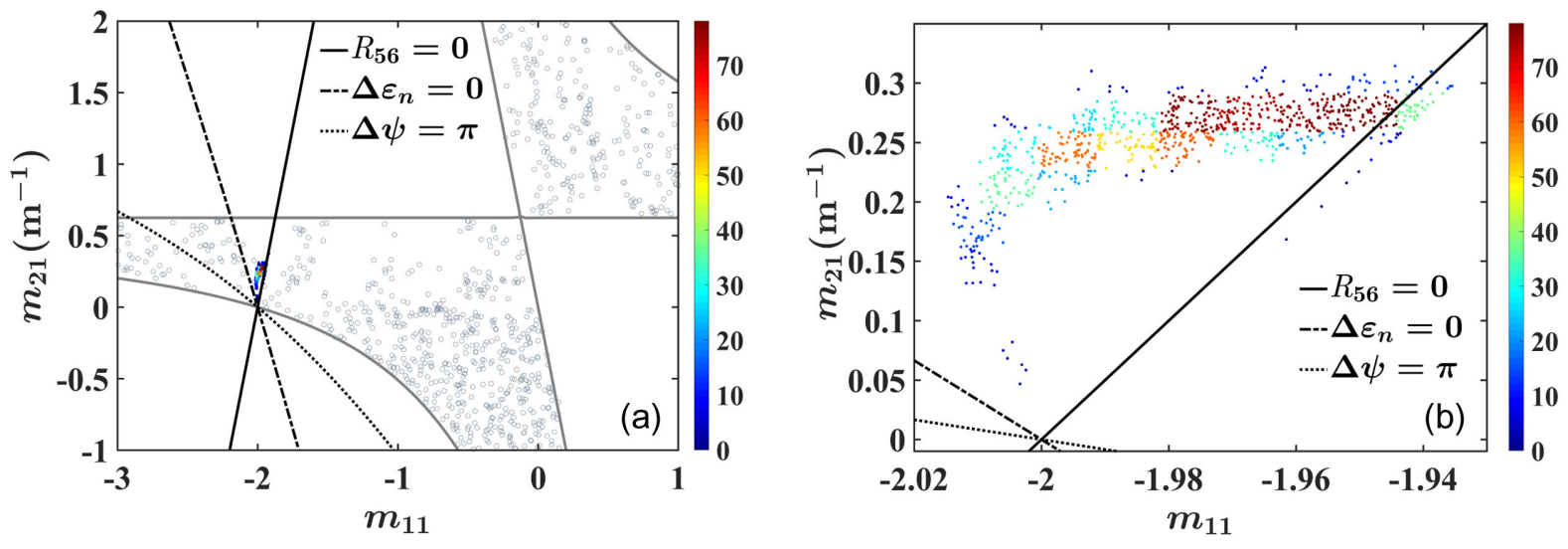

FIG. 6. (a) Distribution of initial seeds (the gray circles), the results of the 2000th generation (dots with color) and the theoretical analysis. (b) Magnified picture of the distribution of the results of the 2000th generation and theoretical analysis. The colors from blue to red represent the distribution of the seeds from sparse to dense. 
TABLE II. The relevant beam and lattice parameters of the two selected solutions.

\begin{tabular}{lcc}
\hline \hline Parameters & $\begin{array}{c}\text { Solution I selected in Case } \\
\text { 1st order isochronous }\end{array}$ & $\begin{array}{c}\text { Solution II selected in Case } \\
\text { high-order isochronous }\end{array}$ \\
\hline$m_{11}$ & -1.996 & -1.904 \\
$m_{21}\left(\mathrm{~m}^{-1}\right)$ & $-6.410 \times 10^{-3}$ & 0.462 \\
$\alpha_{1}$ & $-9.081 \times 10^{-10}$ & $8.952 \times 10^{-7}$ \\
$\alpha_{2}$ & $3.397 \times 10^{-3}$ & $-4.615 \times 10^{-5}$ \\
$\alpha_{3}$ & -0.022 & $-3.869 \times 10^{-3}$ \\
$O\left(\delta^{2}\right)\left[\delta=10^{-3}\right]$ & $3.419 \times 10^{-9}$ & $8.489 \times 10^{-12}$ \\
$O\left(\delta^{2}\right)\left[\delta=10^{-2}\right]$ & $3.620 \times 10^{-7}$ & $4.128 \times 10^{-9}$ \\
$\Delta \varepsilon_{n} / \varepsilon_{n}(\%)$ & 7.740 & 5.898 \\
$\Delta \sigma_{z} / \sigma_{z}(\%)$ & 0.054 & 0.014 \\
$\Delta \psi(\mathrm{rad})$ & 3.162 & 3.157 \\
Total cell length $(\mathrm{m})$ & 10 & 10 \\
\hline \hline
\end{tabular}

new weight factor with the range of $\delta$ being $\left[-10^{-2}, 10^{-2}\right]$. This high-order part is referred to as $O\left(\delta^{2}\right)$, which represents the terms of second order and above in the variable of $\delta$, i.e., $O\left(\delta^{2}\right)=\Delta z / z-\alpha_{1} \delta$, where $\Delta z$ is the variation of the longitudinal path length. The optimized solutions are set to satisfy $\left|\alpha_{1} \delta\right|>2\left|O\left(\delta^{2}\right)\right|$ and we assume that the linear term $\alpha_{1}$ is dominant if this constraint is met. For simplicity, we refer to the optimization case with only $\alpha_{1}$ considered as Case 1st order isochronous. The optimization case with $O\left(\delta^{2}\right)$ suppressed is referred to as Case high-order isochronous.

As a demonstration, we illustrate two lattice solutions. The Solution I, selected in Case 1st order isochronous, is obtained by minimizing $R_{56}$ and the CSR-induced emittance growth. The Solution II, selected in Case high-order isochronous, aims to minimize not only the CSR-induced emittance growth but also the longitudinal dispersion coefficients of the linear and higher orders. The relevant beam and lattice parameters are presented in Table II.
For Solutions I and II, the linear term $\alpha_{1} \delta$ and the residual higher-order part $\Delta z / z-\alpha_{1} \delta$ are shown as a function of $\delta$ in Fig. 7(a). As a representative of the solutions in Case high-order isochronous, the result of Solution II shows that the contributions from high-order longitudinal dispersion functions are flattened compared with the linear term.

Now let us further examine the second and third order longitudinal dispersion functions of the overall solutions we have from the two cases. A fair comparison of the highorder dispersion functions shall be made by keeping smallness of the first order dispersion within a certain level. For this, we randomly select a set of 200 solutions with $\left|\alpha_{1}\right|<1 \times 10^{-5}$ in the two cases. The $\alpha_{2}$ and $\alpha_{3}$ term of the selected solutions are presented in Fig. 7(b), showing a significant difference between the results. The $\alpha_{1}$ terms of these solutions are not shown here as they are similar, but the $\alpha_{2}$ and $\alpha_{3}$ terms are remarkably suppressed when taking the high-order longitudinal dispersion effect into consideration, as shown in Case high-order isochronous.
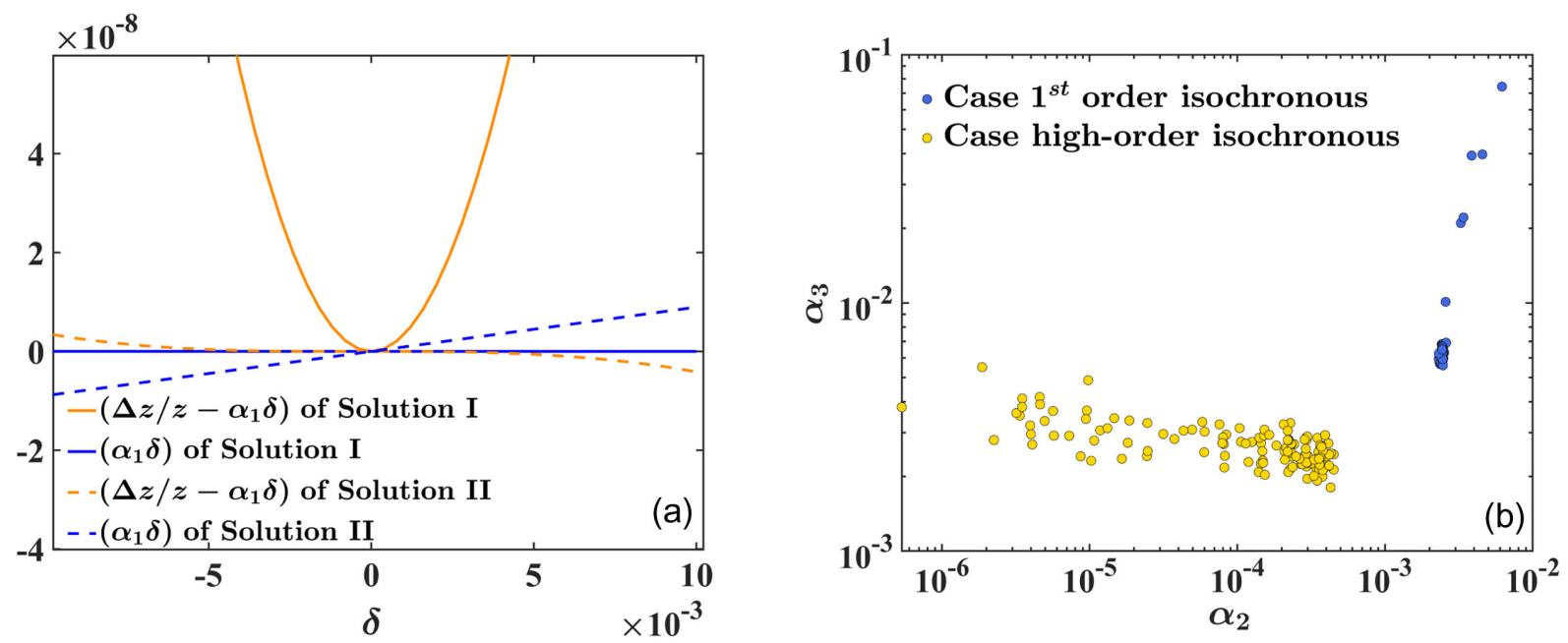

FIG. 7. (a) $\alpha_{1} \delta$ (blue) and $\Delta z / z-\alpha_{1} \delta$ (yellow) of Solution I (solid line) and Solution II (dashed line). (b) The $\alpha_{2}$ and $\alpha_{3}$ term of the selected solutions with $\left|\alpha_{1}\right|<1 \times 10^{-5}$. 
TABLE III. Parameters of the ELEGANT simulation for LPA beams.

\begin{tabular}{lcc}
\hline \hline Parameters & Value & Unit \\
\hline Bunch length & 3 & $\mu \mathrm{m}$ \\
Beam energy & 1500 & $\mathrm{MeV}$ \\
Normalized emittance & 1 & $\mu \mathrm{m} \mathrm{rad}$ \\
Bunch charge & 20 & $\mathrm{pC}$ \\
Relative rms energy spread & 1 & $\%$ \\
\hline \hline
\end{tabular}

This suggests the effectiveness of optimizing the high-order longitudinal dispersion functions as a whole.

\section{B. Simulation results}

\section{Simulation results using conventional rf beam and LPA beam parameters}

The optimizations in Sec. III A are conducted using the conventional rf beam parameters (see Table I). It is worth noticing that in our TBA design, high-order longitudinal dispersion functions are also minimized, which is helpful for transporting beams with large energy spread, e.g., the beam from LPAs. The LPA beams have the characteristics such as large energy spread, e.g., 1\%, ultrashort bunch length on the femtosecond scale, ultralow normalized transverse emittance, and high bunch charge with its energy ranging from a few hundred $\mathrm{MeV}$ to several $\mathrm{GeV}$ [51-55]. To simulate an LPA-like beam, we assume a Gaussian beam in ELEGANT and use the numerical settings presented in Table III. Here we comment that a more realistic LPA beam is not Gaussian and the initial transverse beam properties, i.e., the initial beam size and its divergence, may not directly match the periodic solution of our TBA design. However, it is always possible to manipulate the LPA beam, e.g., by special-purpose high gradient quadrupoles [19] installed upstream of the beam transfer line.

Figure 8 illustrates the results using conventional rf beam and LPA beam parameters of Solutions I and II. The simulation results of $\mathrm{rf}$ beams are also summarized in Table II. For the LPA beams, simulation results show that the relative normalized emittance variation, i.e., $\Delta \varepsilon_{n} / \varepsilon_{n}$ drops from $172.02 \%$ in Solution I to $58.40 \%$ in Solution II. The relative bunch length variation i.e., $\Delta \sigma_{z} / \sigma_{z}$ in Solution II is about one seventh of that in Solution I, which is $76.75 \%$ and $11.21 \%$, respectively. Although the selected solutions in Table II may not be an optimal design for transporting the LPA beams, the simulation results of Solution II have reported considerable mitigation in both CSR-induced emittance growth and bunch length variation.

Moreover, the maintenance of longitudinal phase space is satisfactory. As presented in Figs. 8 and 9, the longitudinal phase space distribution and the current profile are well preserved in both Case 1st order isochronous and Case high-order isochronous for the conventional rf beam transport. However, when using the LPA beam parameters, the longitudinal phase space distribution shown in Fig. 8(e) exhibits severe distortion in Case 1st order isochronous.
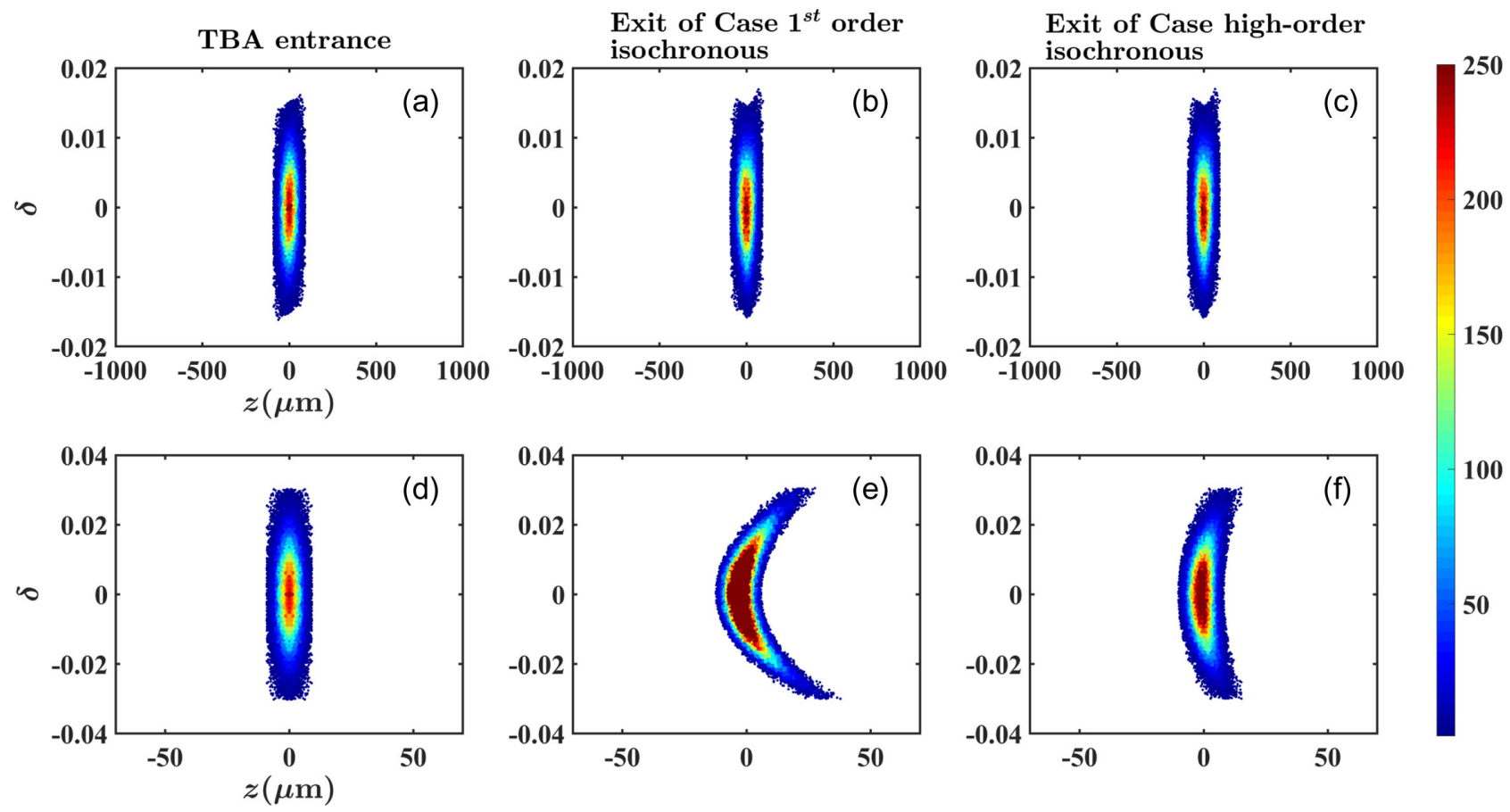

100

50

FIG. 8. Longitudinal phase space distribution of the bunch at the entrance of the TBA (left), the exit of Case 1st order isochronous (middle), and Case high-order isochronous (right), using conventional rf beam parameters [top row, (a), (b) and (c)] and LPA beam parameters [bottom row, (d), (e) and (f)]. The colorbar shows the particle density. 


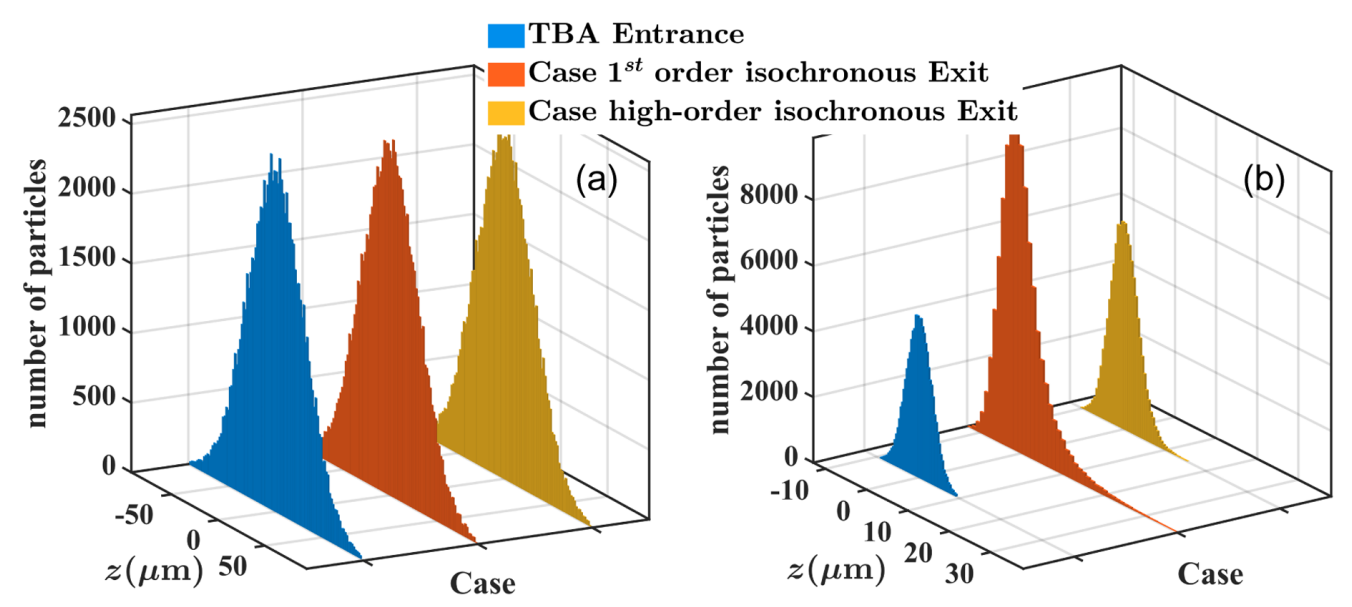

FIG. 9. Comparison of the current prole using conventional rf beam parameters (a) and LPA beam parameters (b).

The current profile in this case deviates from the Gaussian distribution obviously. In contrast, the distortion of the longitudinal phase space distribution is significantly mitigated in Case high-order isochronous.

\section{Simulation results of multiunit cells}

Having studied the beam phase space dynamics in single-cell TBA designs, in this subsection we perform ELEGANT simulations with CSR for a beam transport line by simply concatenating a few of such TBA unit cells. The simulations of our design are based on Solution II (see Table II) and the specific lattice parameters are listed in Table IV. We also use our developed semianalytical tool VOLTERRA [56,57] to evaluate MBI. The results of the semianalytical calculation have been well benchmarked against particle tracking simulations [58,59]. It solves the linearized Vlasov equation for the evolution of the beam microbunching in terms of the phase space microbunching gain. The microbunching gain is characteristic of how the phase space modulation amplifies along a beam transport

TABLE IV. Lattice parameters of Solution II.

\begin{tabular}{lcl}
\hline \hline Parameters & Value & Unit \\
\hline Quadrupole length & 0.2 & $\mathrm{~m}$ \\
Q1 strength & -8.889 & $\mathrm{~m}^{-2}$ \\
Q2 strength & 9.293 & $\mathrm{~m}^{-2}$ \\
Q3 strength & 7.480 & $\mathrm{~m}^{-2}$ \\
Sextupole length & 0.3 & $\mathrm{~m}^{-3}$ \\
SD strength & 321.474 & $\mathrm{~m}^{-3}$ \\
SF strength & -551.934 & $\mathrm{~m}^{-3}$ \\
D1 length & 0.817 & $\mathrm{~m}$ \\
D2 length & 0.331 & $\mathrm{~m}$ \\
D3 length & 0.102 & $\mathrm{~m}$ \\
D4 length & 1.315 & $\mathrm{~m}$ \\
D5 length & 0.125 & $\mathrm{~m}$ \\
D6 length & 0.139 & $\mathrm{~m}$ \\
D7 length & 0.370 & $\mathrm{~m}$ \\
\hline \hline
\end{tabular}

line in the presence of collective effects. Of our particular interest can be the CSR [60,61] and longitudinal space charge (LSC) effect [62]. It is found in our simulation that the MBI caused by LSC is not evident in our designs. So we neglect the LSC effect and consider only the CSR-induced MBI in the following. For the TBA design based on the cross point $\mathrm{A}$, which does not satisfy the stability criterion, it is tested in our simulation that the horizontal beta function, the bunch length, and the normalized transverse emittance exhibit a divergent response for the transfer line consisting of two such TBA cells. For comparison, we build the beam line of our design with two and five periodical TBA cells, and the simulation results using the conventional rf beam parameters (see Table I) are presented in Figs. 10 and 11, respectively.

For our TBA design with stable optics, the optics function, the bunch length, and the normalized transverse emittance, as well as the MBI, are all in good control with two or even five TBA unit cells. The normalized transverse emittance remains to the level of $1 \mu \mathrm{m} \mathrm{rad}$ with two TBA cells and the relative bunch length variation is about $0.06 \%$. The normalized transverse emittance at the exit of five such TBA cells becomes $15 \mu \mathrm{m} \mathrm{rad}$ and the relative bunch length variation is around $0.88 \%$. To quantify MBI in a general transport line, we estimate the microbunching amplification factor $G$ along the beam line. Here $G$ is defined as the modular ratio of the bunching factors at a location $s$ to the initial location $s_{0}$. The microbunching gain at the exit of a beam line is denoted as $G_{f}$ and is a function of the microbunched wavelength $\lambda$. From Figs. 10 and 11, we can see that the maximum value of the microbunching gain in both cases remains around unity, indicating these designs are free from CSR-induced MBI.

It is worth mentioning that Solutions I and II shown in Table II are selected according to the MBI suppression conditions concluded in Ref. [14]. In fact, it is found that the MBI is moderate for only one or two cells even if the phase advance of the adjacent dipoles deviates a little from $\pi$. 

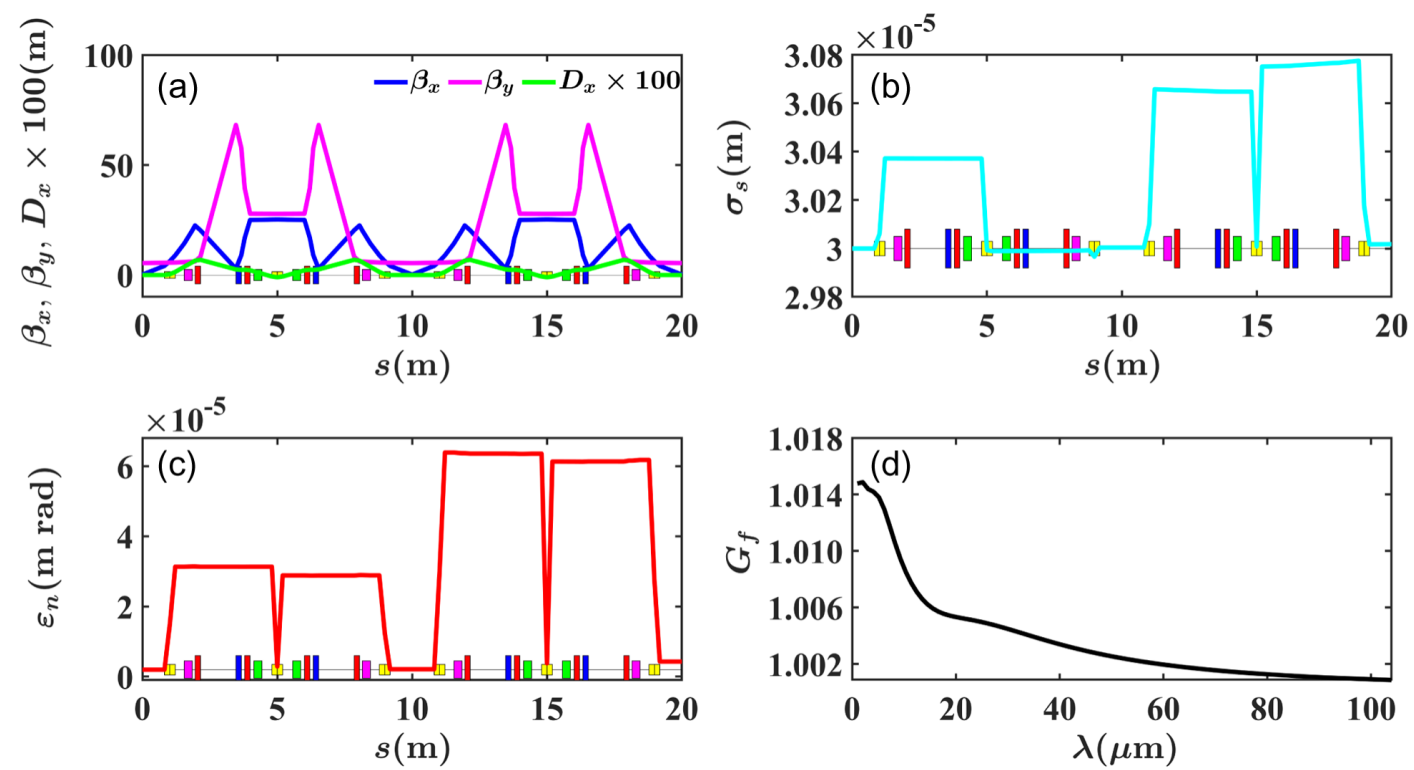

FIG. 10. Simulation results of two TBA cells: (a) optics functions; (b) the bunch length; (c) normalized transverse emittance; (d) the MBI gain.
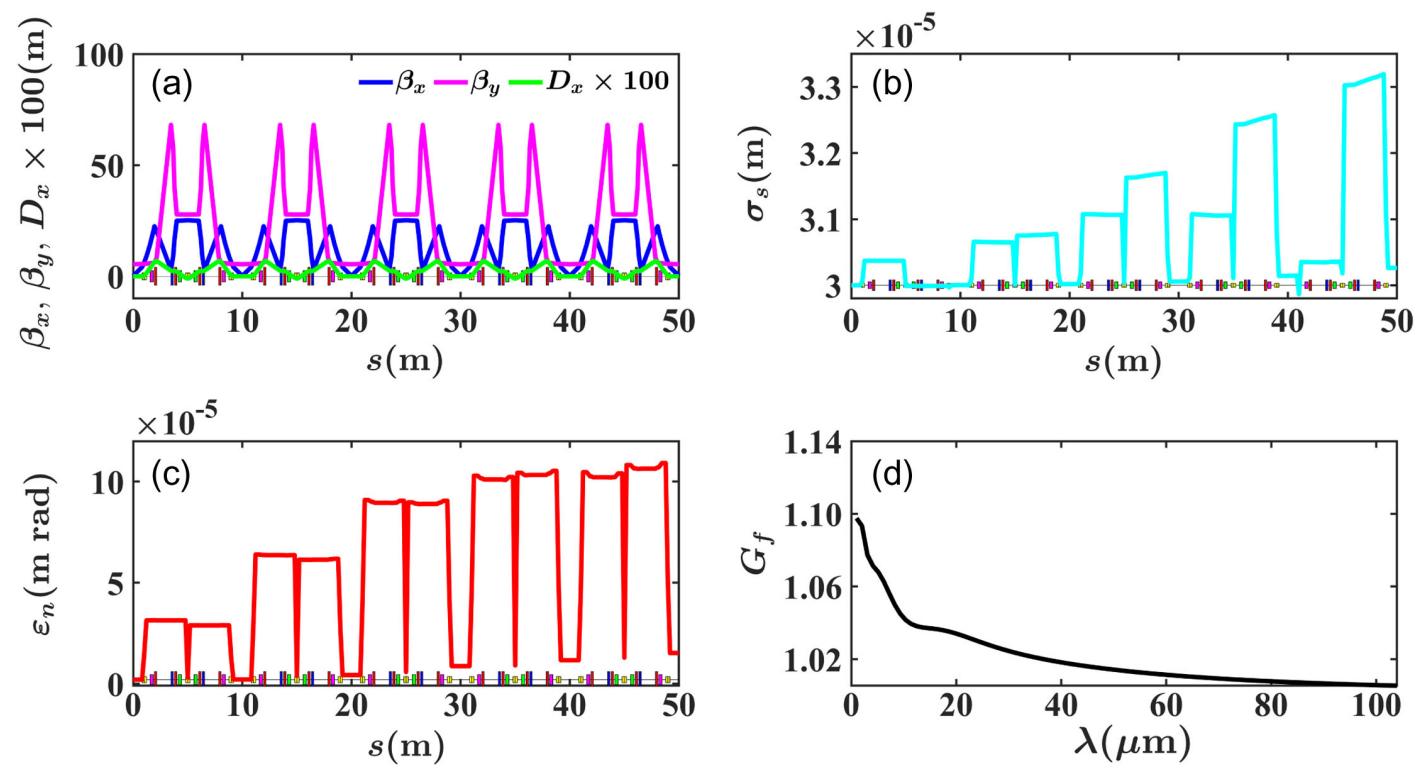

FIG. 11. Simulation results of five TBA cells: (a) optics functions; (b) the bunch length; (c) normalized transverse emittance; (d) the MBI gain.

This means that in the situation where only one or two cells is needed, Solutions I and II may not be optimal choices: smaller values of linear longitudinal dispersion function $\alpha_{1}$ and $\Delta \varepsilon_{n}$ may be reached, meaning that better control of bunch length variation and CSR-induced emittance growth is possible.

\section{CONCLUSION AND DISCUSSION}

In this paper, a theoretical setting of a symmetric TBA cell design with periodic stable optics is identified. The first order longitudinal dispersion and CSR-induced emittance growth can be minimized simultaneously. Considering the periodic stable optics condition, we are forced to strike a compromise between reaching the isochronicity and the cancellation of CSR-induced emittance growth. It has been known that the cross point A which was derived in Ref. [39] does not satisfy the optics stability condition. We find the other solution, the new setting, $\left[m_{11}, m_{21}\left(\mathrm{~m}^{-1}\right)\right]=(-2,0)$. However, this new setting gives a divergent horizontal beta function at the entrance of the TBA cell, since $\left[m_{11}, m_{21}\left(\mathrm{~m}^{-1}\right)\right]=(-2,0)$ is an essential singular point. Despite all that, the solutions near this singular point 
are found to have the potential of being approximately CSR-immune, first-order isochronous and having an extra merit of mitigating the MBI. The MOPSO numerical optimization allows us to design an isochronous cell up to high orders. The TBA design that is approximately CSR immune and isochronous up to high orders, with periodic stable optics and effective suppression of MBI gain, has also been obtained near the new setting. The beam qualities are well preserved for both rf and LPA beams. Such a design strategy points out a new way of lattice design.

In this study, we try to construct as a simple TBA cell as possible in the numerical demonstration tests, where two families of sextupoles are used to obtain unique solutions of the sextupole strengths for specific corrected chromaticities. Nevertheless, one consequence of such a setting is that the natural chromaticity and the corresponding sextupole strengths will change accordingly with the linear optics, and the high-order longitudinal dispersion functions (related to both linear optics design and sextupole strengths) are somewhat correlated to the linear one (depends only on linear optics). Actually, this correlation can be mitigated, by adopting additional families of sextupoles or octupoles in the lattice [12,18,27-30,63]. Following a similar design and optimization procedure as that presented in this paper, it would be feasible to achieve an optimized TBA cell with a better control of the highorder longitudinal dispersion effect.

As for the specific applications of such TBA design, it is primarily oriented towards the single-pass or multipass transfer line for FELs, energy recovery linacs (ERLs), and linear colliders. It is worth noting that careful modifications are needed if such a cell design is applied to storage ring accelerators. As the phase advance of the adjacent dipoles is close to $\pi$, which means that the total phase advance of the TBA is close to $2 \pi$, the optics stability may become more sensitive to the error of the hardware under such a circumstance.

\section{ACKNOWLEDGMENTS}

One of the authors (C. Z.) thanks Biaobin Li, Weihang Liu and Jinyu Wan for discussions. This work is supported by the National Key R\&D Program of China (No. 2016YFA0401900), National Natural Science Foundation of China (No. 11922512), Youth Innovation Promotion Association of Chinese Academy of Sciences (No. Y201904), the Fundamental Research Funds for the Central Universities (HUST) under Project No. 5003131049 and the National Natural Science Foundation of China under Project No. 11905073.

\section{APPENDIX A: DERIVATION OF EQ. (6)}

For completeness, the derivation process for necessary steps and explicit expressions are presented in the Appendix. The entries of total transfer matrix of the TBA cell, $M_{\text {tot }}$, are presented in the following. First, the symplectic condition leads to the constraint $\left(M_{\text {tot }}\right)_{11}\left(M_{\text {tot }}\right)_{22}-\left(M_{\text {tot }}\right)_{12}\left(M_{\text {tot }}\right)_{21}=$ 1 and the achromatic condition by definition requires $\left(M_{\text {tot }}\right)_{16}=0$ and $\left(M_{\text {tot }}\right)_{26}=0$. Then from Eq. (4), Eq. (5) can be obtained. Now the determinant and the explicit expressions of the matrix elements $\left(M_{\mathrm{tot}}\right)$ are

$$
\begin{gathered}
\left(M_{\mathrm{tot}}\right)_{11}\left(M_{\mathrm{tot}}\right)_{22}-\left(M_{\mathrm{tot}}\right)_{12}\left(M_{\mathrm{tot}}\right)_{21}=\left(m_{12} m_{21}-m_{11} m_{22}\right)^{2}, \\
\left(M_{\mathrm{tot}}\right)_{16}=\frac{1}{2} \theta L_{B}^{3} m_{21}^{2}+\frac{1}{2} \theta L_{B}^{2} m_{21}\left(1+2 m_{11}+d m_{21}+3 m_{22}\right)+\theta\left[d+d m_{11}\left(1+m_{22}\right)+m_{12}\left(1+d m_{21}+2 m_{22}\right)\right] \\
+\frac{1}{2} \theta L_{B}\left[1+m_{22}+2 m_{22}^{2}+m_{11}\left(2+2 d m_{21}+3 m_{22}\right)+m_{21}\left(d+3 m_{12}+2 d m_{22}\right)\right], \\
\left(M_{\mathrm{tot}}\right)_{26}=\frac{1}{2} \theta\left[\left(2+2 m_{12} m_{21}+L_{B}^{2} m_{21}^{2}\right)+2 m_{11}\left(1+L_{B} m_{21}+m_{22}\right)+L_{B} m_{21}\left(1+2 m_{22}\right)\right], \\
\left(M_{\mathrm{tot}}\right)_{56}=\frac{1}{4} \theta^{2}\left[L_{B}^{3} m_{21}^{2}+8 m_{12}\left(1+m_{22}\right)+2 L_{B}^{2} m_{21}\left(1+m_{11}+2 m_{22}\right)\right] \\
+\frac{1}{4} \theta^{2}\left\{L_{B}\left[2+4 m_{12} m_{21}+4 m_{22}+4 m_{22}^{2}+4 m_{11}\left(1+m_{22}\right)\right]\right\} .
\end{gathered}
$$

With the imposition of the first-order isochronous condition, i.e., $\left(M_{\text {tot }}\right)_{56}=0$, we can derive Eq. (6).

\section{APPENDIX B: CSR KICK MODEL AND DERIVATION OF EQ. (7)}

The CSR kick model [25] provides a way to evaluate the CSR-induced emittance growth during evolution of the particle motion in the 2D $\left(x, x^{\prime}\right)$ plane. The effect of CSR in an $n$-dipole achromat can be expressed explicitly with the analysis of $n$-point kicks separately, which greatly simplifies the analysis and can be conveniently applied to the numerical optimizations for the CSRimmune lattice design. In the model, the CSR-induced projected emittance growth in the dipole magnet can be estimated by 


$$
\begin{aligned}
\Delta \varepsilon_{n} & =\varepsilon_{n}-\varepsilon_{n 0} \approx \frac{1}{2} \gamma \beta d \varepsilon, \\
d \varepsilon & =\gamma_{1 x} x_{k}^{2}+2 \alpha_{1 x} x_{k} x^{\prime}{ }_{k}+\beta_{1 x} x_{k}^{\prime}{ }_{k}^{2},
\end{aligned}
$$

where $\gamma$ is the Lorentz factor, $\beta$ is the particle velocity relative to the speed of light, $\alpha_{1 x}, \beta_{1 x}$, and $\gamma_{1 x}$ are the Twiss parameters at the exit of the dipole. The net CSRinduced coordinate deviations exerted by the dipole are given by

$X_{k}=\left(\begin{array}{c}x_{k} \\ x^{\prime}{ }_{k}\end{array}\right)=\left(\begin{array}{c}\rho^{4 / 3} k[\theta \cos (\theta / 2)-2 \sin (\theta / 2)] \\ \sin (\theta / 2)\left(2 \delta+\rho^{1 / 3} \theta k\right)\end{array}\right)$,

where $\rho$ and $\theta$ are the radius and angle of the magnet, and $\delta=\delta_{0}+\delta_{\text {csr }}$ is the energy deviation, with $\delta_{0}$ being the initial energy deviation. The effect of CSR is equivalently formulated with a point kick occurring at the center of each dipole, which leads to the coordinate deviations [Eq. (B2)]. The energy deviation $\delta$ is increased by $\delta_{\text {csr }}=k L_{B} / \rho^{2 / 3}$, after passing through one single dipole magnet. The parameter $k$ is

$$
k=0.2459 \frac{e Q \mu_{0} c_{0}^{2}}{4 \pi E \sigma_{z}^{4 / 3}},
$$

with $e$ being the charge of a single particle, $Q$ being the bunch charge, $\mu_{0}$ being the permeability of vacuum, $c_{0}$ being the speed of light, $E$ being the beam energy and $\sigma_{z}$ being the rms bunch length.

For the TBA with three identical dipoles, the particle coordinates relative to the reference trajectory can be obtained as follows. The cancellation condition of the CSR-induced emittance growth has been derived in Ref. [26] [see Eq. (15) in Ref. [26] ]. Here we just replay some important derivation steps. At the center of the first dipole, the particle experiences the first CSR kick and the coordinates are given by

$$
X_{1}=X_{0}+X_{k 1},
$$

where $X_{0}$ is the initial particle coordinates. Here we define the transfer matrix from the center of the first dipole to the center of the second dipole as $M_{c 2 c}$, which can be expressed as

$$
M_{c 2 c}=R_{B h} M R_{B h}
$$

where $R_{B h}$ is the transfer matrix of half of the dipole magnet. Then after the particle passing through the section from the center of the first dipole to that of the second dipole, the particle experiences the second CSR kick and the coordinates can be expressed as

$$
X_{2}=M_{c 2 c} X_{1}+X_{k 2}
$$

Similarly, after the beam acquires the third CSR kick and the coordinates are given by

$$
X_{3}=M_{c 2 c}^{*} X_{2}+X_{k 3},
$$

with $M_{c 2 c}^{*}=R_{B h} \hat{M} R_{B h}$. The energy deviations now become $\rho^{1 / 3} \theta k+\delta_{0}$ and $2 \rho^{1 / 3} \theta k+\delta_{0}$ in $X_{k 2}$ and $X_{k 3}$, respectively.

The cancellation condition of the CSR-induced emittance growth requires that $X_{3}=(0,0)^{T}$, having

$$
\left(M_{c 2 c}\right)_{11}+\frac{2 \theta \sin \left(\frac{\theta}{2}\right)}{\left[2 \sin \left(\frac{\theta}{2}\right)-\theta \cos \left(\frac{\theta}{2}\right)\right] \frac{L_{B}}{\theta}}\left(M_{c 2 c}\right)_{12}=-\frac{1}{2} .
$$

We adopt the small-angle approximation in which $\theta \ll 1$ and the CSR-induced emittance growth cancellation condition can be simplified as

$$
\left(M_{c 2 c}\right)_{11}+\frac{12}{L_{B}}\left(M_{c 2 c}\right)_{12}=-\frac{1}{2}
$$

We substitute the explicit expressions of $M_{c 2 c}$ which can be obtained from Eq. (B5), to the CSR-induced emittance growth cancellation condition, i.e., Eq. (B9), then Eq. (7) can be derived.

\section{APPENDIX C: EXPLICIT EXPRESSIONS FOR THE PERIODIC TWISS FUNCTIONS}

The Twiss functions at the entrance and the exit of the TBA can be related by

$$
\left(\begin{array}{c}
\beta_{x f} \\
\alpha_{x f} \\
\gamma_{x f}
\end{array}\right)=\left(\begin{array}{ccc}
\left(M_{\mathrm{tot}}\right)_{11}^{2} & -2\left(M_{\mathrm{tot}}\right)_{11}\left(M_{\mathrm{tot}}\right)_{12} & \left(M_{\mathrm{tot}}\right)_{12}^{2} \\
-\left(M_{\mathrm{tot}}\right)_{11}\left(M_{\mathrm{tot}}\right)_{21} & \left(M_{\mathrm{tot}}\right)_{11}\left(M_{\mathrm{tot}}\right)_{22}+\left(M_{\mathrm{tot}}\right)_{12}\left(M_{\mathrm{tot}}\right)_{21} & -\left(M_{\mathrm{tot}}\right)_{12}\left(M_{\mathrm{tot}}\right)_{22} \\
\left(M_{\mathrm{tot}}\right)_{21}^{2} & -2\left(M_{\mathrm{tot}}\right)_{21}\left(M_{\mathrm{tot}}\right)_{22} & \left(M_{\mathrm{tot}}\right)_{22}^{2}
\end{array}\right)\left(\begin{array}{l}
\beta_{x 0} \\
\alpha_{x 0} \\
\gamma_{x 0}
\end{array}\right),
$$

where $\beta_{x 0}, \alpha_{x 0}$ and $\gamma_{x 0}$ are the Twiss functions at the entrance of the TBA and $\beta_{x f}, \alpha_{x f}$ and $\gamma_{x f}$ are the Twiss functions at the exit. For periodic Twiss functions, we have $\beta_{x f}=\beta_{x 0}, \alpha_{x f}=\alpha_{x 0}$ and $\gamma_{x f}=\gamma_{x 0}$. Here we present the explicit expressions for the periodic Twiss functions of TBA structure. Also, the periodic Twiss functions can be expressed in terms of $m_{11}$ and $m_{21}$ with the adoption of the symplectic condition and the achromatic condition: 


$$
\begin{aligned}
& \beta_{x 0}=\frac{\sqrt{\left(-1+2 d m_{21}+L_{B} m_{21}\right)\left[-4+L_{B}^{2} m_{21}^{2}+L_{B} m_{21}\left(-1+2 d m_{21}\right)+2 m_{11}\left(-1+2 d m_{21}+L_{B} m_{21}\right)\right]}}{2 \sqrt{-m_{21}^{2}\left(2 m_{11}+L_{B} m_{21}\right)}}, \\
& \alpha_{x 0}=0 \\
& \gamma_{x 0}=1 / \beta_{x 0} .
\end{aligned}
$$

Here one can get that, for the cross point B derived in the above, where $m_{21}=0$ and $m_{11}=-2$, we have $\beta_{x 0} \propto 1 / \sqrt{m_{21}}$ as $m_{21} \rightarrow 0^{+}$. It means that the $\beta_{0}$ function will indeed diverge at the cross point $\mathrm{B}$.

We revalidate the feasibility of the cross point B using the analytical method reported in Ref. [39]. The horizontal emittance at the exit of the beam line, $\varepsilon_{x}$ reads [see Eq. (12) in Ref. [39] ]

$$
\varepsilon_{x}^{2}=\varepsilon_{x 0}^{2}+\varepsilon_{x 0}\left(\beta_{x f}\left\langle\Delta x^{\prime 2}\right\rangle+2 \alpha_{x f}\left\langle\Delta x \Delta x^{\prime}\right\rangle+\gamma_{x f}\left\langle\Delta x^{2}\right\rangle\right)+\Delta \varepsilon_{x}^{2},
$$

where $\varepsilon_{x 0}$ is the unperturbed emittance, $\Delta x$ is the CSR induced coordinate deviation and $\Delta x^{\prime}$ is the derivative. The notation \langle\rangle means averaging over the bunch population in phase space and

$$
\Delta \varepsilon_{x}^{2}=\left\langle\Delta x^{2}\right\rangle\left\langle\Delta x^{\prime 2}\right\rangle-\left\langle\Delta x \Delta x^{\prime}\right\rangle^{2},
$$

which is zero under the assumption that the longitudinal beam density remains stationary. It is found that, with $\beta_{x 0}\left\langle\Delta x^{\prime 2}\right\rangle \propto m_{21}{ }^{3 / 2} \rightarrow 0, \alpha_{x 0}=0$, and $\gamma_{x 0}\left\langle\Delta x^{2}\right\rangle \rightarrow 0$ (since $\gamma_{x 0}=1 / \beta_{x 0}$ and $\left\langle\Delta x^{2}\right\rangle$ is finite), we have $\beta_{x f}\left\langle\Delta x^{\prime 2}\right\rangle+2 \alpha_{x f}\left\langle\Delta x \Delta x^{\prime}\right\rangle+\gamma_{x f}\left\langle\Delta x^{2}\right\rangle \rightarrow 0$, yielding minimized emittance growth.

From this perspective, i.e., aiming at minimizing the Twiss-functions' mismatch induced by CSR, one can always get the solutions using a set of equations, i.e., $\beta_{x 0}=$ 0 and $\left(M_{\text {tot }}\right)_{56}=0$ (which is referred to as Case BETA) or using $\gamma_{x 0}=0$ and $\left(M_{\text {tot }}\right)_{56}=0$ (which is referred to as Case GAMMA). In Case BETA, we get another two solutions,

$$
\begin{array}{ll}
m_{11}=\frac{-8 d-3 L_{B}}{2\left(2 d+L_{B}\right)}, & m_{21}=\frac{1}{2 d+L_{B}}, \\
m_{11}=\frac{-4 d-L_{B}}{2\left(2 d+L_{B}\right)}, & m_{21}=\frac{4 d+3 L_{B}}{L_{B}\left(2 d+L_{B}\right)},
\end{array}
$$

and in Case GAMMA, we get another solution,

$$
m_{11}=-1, \quad m_{21}=\frac{2}{L_{B}} .
$$

We have also analyzed these derived solutions. However, they do not give satisfying results on suppressing the emittance growth.

[1] L.-H. Yu, M. Babzien, I. Ben-Zvi, L. F. DiMauro, A. Doyuran, W. Graves et al., High-gain harmonic-generation free-electron laser, Science 289, 932 (2000).

[2] W. Ackermann, G. Asova, V. Ayvazyan, A. Azima, N. Baboi, J. Bähr et al., Operation of a free-electron laser from the extreme ultraviolet to the water window, Nat. Photonics 1, 336 (2007).

[3] T. Ishikawa, H. Aoyagi, T. Asaka, Y. Asano, N. Azumi, T. Bizen et al., A compact X-ray free-electron laser emitting in the sub-Ångström region, Nat. Photonics 6, 540 (2012).

[4] J. Feldhaus, E. L. Saldin, E. A. Schneidmiller, and M. V. Yurkov, Photon ring multiuser distribution system for soft X-ray SASE FEL laboratory, Nucl. Instrum. Methods Phys. Res., Sect. A 507, 510 (2003).

[5] E. Allaria, D. Castronovo, P. Cinquegrana, P. Craievich, M. Dal Forno, M. B. Danailov et al., Two-stage seeded soft-Xray free-electron laser, Nat. Photonics 7, 913 (2013).

[6] W. S. Graves, J. Bessuille, P. Brown, S. Carbajo, V. Dolgashev, K.-H. Hong et al., Compact X-ray source based on burst-mode inverse Compton scattering at $100 \mathrm{kHz}$, Phys. Rev. ST Accel. Beams 17, 120701 (2014).

[7] K. Dupraz, K. Cassou, N. Delerue, P. Fichot, A. Martens, A. Stocchi et al., Design and optimization of a highly efficient optical multipass system for $\gamma$-ray beam production from electron laser beam Compton scattering, Phys. Rev. ST Accel. Beams 17, 033501 (2014).

[8] V. Petrillo, A. Bacci, C. Curatolo, M. Ferrario, G. Gatti, C. Maroli et al., Dual color x rays from Thomson or Compton sources, Phys. Rev. ST Accel. Beams 17, 020706 (2014).

[9] B. Faatz, E. Plönjes, S. Ackermann, A. Agababyan, V. Asgekar, V. Ayvazyan et al., Simultaneous operation of two soft x-ray free-electron lasers driven by one linear accelerator, New J. Phys. 18, 062002 (2016).

[10] J. A. G. Akkermans, S. Di Mitri, D. Douglas, and I. D. Setija, Compact compressive arc and beam switchyard for energy recovery linac-driven ultraviolet free electron lasers, Phys. Rev. Accel. Beams 20, 080705 (2017).

[11] S. Di Mitri and S. Spampinati, Microbunching instability study in a linac-driven free electron laser spreader beam line, Phys. Rev. Accel. Beams 20, 120701 (2017).

[12] W. A. Gillespie, An FEL beam injection system with adjustable isochronism, Nucl. Instrum. Methods Phys. Res., Sect. A 374, 395 (1996).

[13] C.-Y. Tsai, Suppressing CSR microbunching in recirculation arcs, in Proceedings of the 9th International Particle Accelerator Conference (IPAC 2018), Vancouver, BC, Canada (JACOW, Geneva, Switzerland, 2018), pp. 1784-1789.

[14] C.-Y. Tsai, S. Di Mitri, D. Douglas, R. Li, and C. Tennant, Conditions for coherent-synchrotron-radiation-induced 
microbunching suppression in multibend beam transport or recirculation arcs, Phys. Rev. Accel. Beams 20, 024401 (2017).

[15] Y.S. Derbenev, J. Rossbach, E. L. Saldin, and V. D. Shiltsev, Microbunch radiative tail-head interaction, Report No. TESLA-FEL 95-05.

[16] S. Heifets and G. Stupakov, Beam instability and microbunching due to coherent synchrotron radiation, Report No. SLAC-PUB-9425 03.

[17] S. Seletskiy, Y. Hidaka, J. B. Murphy, B. Podobedov, H. Qian, Y. Shen, X. J. Wang, and X. Yang, Initial source of microbunching instability studies in a free electron laser injector, Phys. Rev. ST Accel. Beams 14, 110701 (2011).

[18] R. J. England, J. B. Rosenzweig, G. Andonian, P. Musumeci, G. Travish, and R. Yoder, Sextupole correction of the longitudinal transport of relativistic beams in dispersionless translating sections, Phys. Rev. ST Accel. Beams 8, 012801 (2005).

[19] T. Liu, T. Zhang, D. Wang, and Z. Huang, Compact beam transport system for free-electron lasers driven by a laser plasma accelerator, Phys. Rev. Accel. Beams 20, 020701 (2017).

[20] D. Douglas, Suppression, and enhancement of CSRdriven emittance degradation in the IR-FEL driver, Thomas Jefferson National Accelerator Facility Report No. JLABTN-98-012, 1998.

[21] S. Di Mitri, M. Cornacchia, and S. Spampinati, Cancellation of Coherent Synchrotron Radiation Kicks with Optics Balance, Phys. Rev. Lett. 110, 014801 (2013).

[22] D. Douglas, S. V. Benson, A. Hutton, G. A. Krafft, R. Li, G. R. Neil, Y. Roblin, C. D. Tennant, and C.-Y. Tsai, Control of coherent synchrotron radiation and microbunching effects during transport of high brightness electron beams, http://arxiv.org/abs/1403.2318.

[23] D. R. Douglas, S. V. Benson, R. Li, Y. Roblin, and C. D. Tennant, Control of synchrotron radiation effects during recirculation with bunch compression, in Proceedings of the 6th International Particle Accelerator Conference (IPAC 2015), Richmond, VA (JACOW, Geneva, Switzerland, 2015), pp. 1910-1912.

[24] R. Hajima, A first-order matrix approach to the analysis of electron beam emittance growth caused by coherent synchrotron radiation, Jpn. J. Appl. Phys. 42, L974 (2003).

[25] Y. Jiao, X. Cui, X. Huang, and G. Xu, Generic conditions for suppressing the coherent synchrotron radiation induced emittance growth in a two-dipole achromat, Phys. Rev. ST Accel. Beams 17, 060701 (2014).

[26] X.-Y. Huang, Y. Jiao, G. Xu, and X.-H. Cui, Suppression of the emittance growth induced by coherent synchrotron radiation in triple-bend achromats, Chin. Phys. C 39, 057001 (2015).

[27] A. Sharma, Abdurrahim, A. D. Ghodke, and G. Singh, Optics design and second order longitudinal dispersion minimization in a bunch compressor transfer line for CTF3, Nucl. Instrum. Methods Phys. Res., Sect. A 602, 342 (2009).

[28] S. Thorin, F. Curbis, M. Eriksson, O. Karlberg, S. Werin, D. Angal-Kalinin, and P. Williams, Study of some design concepts and collective effects in the MAX IV linac, in
Proceedings of the 2nd International Particle Accelerator Conference (IPAC 2011), San Sebastián, Spain (JACOW, Geneva, Switzerland, 2011), pp. 3176-3178.

[29] A. Jackson, A comparison of the Chasman-Green and triple bend achromat lattices, Part. Accel. 22, 111 (1987).

[30] R. Hajima, Optics and beam transport in energy-recovery linacs, Nucl. Instrum. Methods Phys. Res., Sect. A 557, 45 (2006).

[31] E. L. Saldin, E. A. Schneidmiller, and M. V. Yurkov, Longitudinal space charge-driven microbunching instability in the TESLA Test Facility linac, Nucl. Instrum. Methods Phys. Res., Sect. A 528, 355 (2004).

[32] Z. Huang, M. Borland, P. Emma, J. Wu, C. Limborg, G. Stupakov, and J. Welch, Suppression of microbunching instability in the linac coherent light source, Phys. Rev. ST Accel. Beams 7, 074401 (2004).

[33] C. Behrens, Z. Huang, and D. Xiang, Reversible electron beam heating for suppression of microbunching instabilities at free-electron lasers, Phys. Rev. ST Accel. Beams 15, 022802 (2012).

[34] J. Qiang, C. E. Mitchell, and M. Venturini, Suppression of Microbunching Instability Using Bending Magnets in Free-Electron-Laser Linacs, Phys. Rev. Lett. 111, 054801 (2013).

[35] S. Di Mitri and S. Spampinati, Microbunching Instability Suppression via Electron-Magnetic-Phase Mixing, Phys. Rev. Lett. 112, 134802 (2014).

[36] G. Vignola, Preliminary design of a dedicated $6 \mathrm{GeV}$ synchrotron radiation storage ring, Nucl. Instrum. Methods Phys. Res., Sect. A 236, 414 (1985).

[37] P. Emma and R. Brinkmann, Emittance dilution through coherent energy spread generation in bending system, in Proceedings of the Particle Accelerator Conference (PAC 1997), Vancouver, BC, Canada (JACOW, Geneva, Switzerland, 1997), pp. 1679-1681.

[38] M. Venturini, CSR-induced emittance growth in achromats: Linear formalism revisited, Nucl. Instrum. Methods Phys. Res., Sect. A 794, 109 (2015).

[39] M. Venturini, Design of a triple-bend isochronous achromat with minimum coherent-synchrotron-radiationinduced emittance growth, Phys. Rev. Accel. Beams 19, 064401 (2016).

[40] C. A. Coello Coello and M. S. Lechuga, MOPSO: A proposal for multiple objective particle swarm optimization, in Proceedings of the 2002 Congress on Evolutionary Computation (CEC'02), Honolulu, HI, USA (IEEE, Piscataway, NJ, 2002), pp. 1051-1056.

[41] R. Akbari and K. Ziarati, A rank based particle swarm optimization algorithm with dynamic adaptation, J. Comput. Appl. Math. 235, 2694 (2011).

[42] F. Marini and B. Walczak, Particle swarm optimization (PSO). A tutorial, Chemom. Intell. Lab. Syst. 149, 153 (2015).

[43] J. Li, J. Feikes, P. Goslawski, and M. Ries, Particle swarm optimization algorithm applied in online commissioning at the MLS and BESSY II, in Proceedings of the 8th International Particle Accelerator Conference (IPAC 2017), Copenhagen, Denmark (JACOW, Geneva, Switzerland, 2017), pp. 3700-3703. 
[44] A. Tharwat and W. Schenck, A conceptual and practical comparison of PSO-style optimization algorithms, Expert Syst. Appl. 167, 114430 (2021).

[45] X. Pang and L. J. Rybarcyk, Multiobjective particle swarm and genetic algorithm for the optimization of the LANSCE linac operation, Nucl. Instrum. Methods Phys. Res., Sect. A 741, 124 (2014).

[46] X. Huang and J. Safranek, Nonlinear dynamics optimization with particle swarm and genetic algorithms for SPEAR3 emittance upgrade, Nucl. Instrum. Methods Phys. Res., Sect. A 757, 48 (2014).

[47] Y. Jiao and G. Xu, Optimizing the lattice design of a diffraction-limited storage ring with a rational combination of particle swarm and genetic algorithms, Chin. Phys. C 41, 027001 (2017).

[48] J. Xu, P. Yang, G. Liu, Z. Bai, and W. Li, Constraint handling in constrained optimization of a storage ring multibend-achromat lattice, Nucl. Instrum. Methods Phys. Res., Sect. A 988, 164890 (2021).

[49] M. Borland, Simple method for particle tracking with coherent synchrotron radiation, Phys. Rev. ST Accel. Beams 4, 070701 (2001).

[50] K. Y. Ng, Momentum compaction and phase slip factor, Fermi National Accelerator Laboratory Report No. FERMILAB-FN-0900-APC, 2010.

[51] C. B. Schroeder, E. Esarey, W. P. Leemans, J. van Tilborg, F. Grüner, A. R. Maier, Y. Ding, and Z. Huang, Freeelectron lasers driven by laser-plasma accelerators using decompression or dispersion, in Proceedings of the 35th International Free Electron Laser Conference (FEL 2013), Manhattan, NY (JACOW, Geneva, Switzerland, 2013), pp. 117-121.

[52] M. Khojoyan, F. Briquez, M. Labat, A. Loulergue, O. Marcouillé, F. Marteau, G. Sharma, and M. E. Couprie, Transport studies of LPA electron beam towards the FEL amplification at COXINEL, Nucl. Instrum. Methods Phys. Res., Sect. A 829, 260 (2016).

[53] M. E. Couprie, M. Labat, C. Evain, F. Marteau, F. Briquez, M. Khojoyan et al., An application of laser-plasma acceleration: Towards a free-electron laser amplification, Plasma Phys. Controlled Fusion 58, 034020 (2016).
[54] T. Liu, T. Zhang, B. Liu, D. Wang, Z. Huang, and J. Liu, Beam transport line of the LPA-FEL facility based on transverse gradient undulator, in Proceedings of the 7th International Particle Accelerator Conference (IPAC 2016): Busan, Korea (JACOW, Geneva, Switzerland, 2016), pp. 3287-3290.

[55] M. Labat, S. Bielawski, A. Loulergue, S. Corde, M.-E. Couprie, and E. Roussel, Interferometry for full temporal reconstruction of laser-plasma accelerator-based seeded free electron lasers, New J. Phys. 22, 013051 (2020).

[56] C.-Y. Tsai, D. Douglas, R. Li, and C. Tennant, Linear microbunching analysis for recirculation machines, Phys. Rev. Accel. Beams 19, 114401 (2016).

[57] C.-Y. Tsai, Y.-S. Derbenev, D. Douglas, R. Li, and C. Tennant, Vlasov analysis of microbunching instability for magnetized beams, Phys. Rev. Accel. Beams 20, 054401 (2017).

[58] C.-Y. Tsai and R. Li, Simulation of coherent synchrotron radiation induced microbunching gain using ELEGANT, Technical Report No. JLAB-TN-14-016, 2016.

[59] C.-Y. Tsai, Investigation of microbunching instabilities in modern recirculating accelerators, Technical Report No. JLAB-ACC-17-2439, Thomas Jefferson National Accelerator Facility, Newport News, VA, 2017, http://hdl .handle.net/10919/77429.

[60] R. Li and C.-Y. Tsai, CSR impedance for nonultrarelativistic beams, in Proceedings of the 6th International Particle Accelerator Conference (IPAC 2015), Richmond, VA (JACOW, Geneva, Switzerland, 2015), pp. 709-712.

[61] R. Li and C.-Y. Tsai, Entrance and exit CSR impedance for nonultrarelativistic beam, in Proceedings of the 8th International Particle Accelerator Conference (IPAC 2017), Copenhagen, Denmark (JACOW, Geneva, Switzerland, 2017), pp. 3214-3217.

[62] M. Venturini, Models of longitudinal space-charge impedance for microbunching instability, Phys. Rev. ST Accel. Beams 11, 034401 (2008).

[63] J. B. Svensson, T. K. Charles, O. Lundh, and S. Thorin, Third-order double-achromat bunch compressors for broadband beams, Phys. Rev. Accel. Beams 22, 104401 (2019). 